\title{
Formation Tracking for High-Order Time-Invariant Swarm Systems with Limited Energy and Fixed Topologies
}

\author{
Jianye Yang $\left(\mathbb{D},{ }^{1}\right.$ Cheng Wang $\left(\mathbb{D},{ }^{2}\right.$ Hongtao Dang $\mathbb{D},{ }^{1}$ and Xinzhong Han $\mathbb{D}^{3}$ \\ ${ }^{1}$ School of Science, Xijing University, Xi'an 710123, China \\ ${ }^{2}$ High-Tech Institute of Xi'an, Xi'an 710025, China \\ ${ }^{3}$ Beijing BlueVision Technology Limited Company, Beijing 100070, China \\ Correspondence should be addressed to Cheng Wang; m15212783833@163.com
}

Received 3 October 2020; Accepted 13 October 2020; Published 28 October 2020

Academic Editor: Ning Cai

Copyright (๑) 2020 Jianye Yang et al. This is an open access article distributed under the Creative Commons Attribution License, which permits unrestricted use, distribution, and reproduction in any medium, provided the original work is properly cited.

The current paper investigates design and analysis problems of formation tracking for high-order linear time-invariant swarm systems, where communication topologies among agents have leader-following structures and the whole energy supply is limited. Firstly, the communication topology of a swarm system is depicted by a directed graph with a spanning tree, where the communication channels from the leader to followers are directional and the communication channels among followers are bidirectional, and a new formation tracking protocol with an energy integral term and a given upper bound is proposed to achieve formation tracking with the limited energy. Then, sufficient conditions for time-varying formation tracking design and analysis with the limited energy are presented, respectively, which include two/three linear matrix inequality constraints associated with the maximum and minimum nonzero eigenvalues of the Laplacian matrix of a communication topology. Especially, time-invariant formation tracking criteria are further deduced. Finally, two numerical examples are revealed to verify main theoretical conclusions.

\section{Introduction}

In the last decade, many scientists paid their attentions to design different cooperative control strategies of swarm systems with different application backgrounds, such as explanations for animal flocking phenomena [1, 2], information consensus analysis and design [3-9], analysis for different source data [10], and formation structure design and maintenance [10-13]. Distributed formation is inspired by the biological formation flying without the superintend vertex, where the formation structure maintenance is realized via distributed information transmissions and local interactions. It should be pointed out that distributed formation control has extensive applications in the cooperative operation of satellite clusters, the coordinated assembly of multiple robots, and the collaborative attack of multiple unmanned aerial ships, and Ren [14] summarized the existing formation control approaches including the virtualstructure one, the behavior-based one, and the consensus-based one and pointed out that the consensus-based approach can achieve distributed formation control and can develop the precise mathematical analysis. The consensus-based formation of linear swarm systems as well as their controllability was discussed in [15-17].

Based on the time-varying characteristics of formation structures, distributed formation can be divided into timevarying distributed formation and time-invariant distributed formation. For time-varying distributed formation control, the formation geometric structure is time-varying even if the formation structure is formed. For time-invariant distributed formation control, the formation geometric structure is timeinvariant once the formation structure is finished. By using the Nyquist stability criterion, time-invariant formation criteria for swarm systems were proposed in [18], where the communication topology was modeled by graph theory and several important features of the communication topology were shown. By a nonlinear formation protocol, sufficient conditions for finite-time time-invariant formation were revealed in [19], 
where theoretical results were applied into multiple nonholonomic robot systems. Qin et al. [20] studied the impacts of intermittent communications on time-invariant formation control for swarm systems with time delays. In [21-23], sufficient and/or necessary conditions of time-varying formation design and analysis were presented, where it was shown that time-varying formation control is more challenging than timeinvariant formation control and formation feasible conditions of the time-varying formation are more complex than formation feasible conditions of the time-invariant formation.

According to communication topology structure properties among agents, distributed formation can be divided into two types: the leaderless distributed formation and the leader-following distributed formation. In the leaderless distributed formation, all agents in a swarm system have the same decision weight as shown in [18-23]. The leader-following distributed formation is also called formation tracking, where the leader plays a role similar to the superintend vertex and all following vertexes track the leader in a specific formation structure. For first-order swarm systems, Xiao et al. [24] proposed some novel sufficient conditions for formation tracking, where each agent was described as a first-order integrator, which means that the state of one agent is not time-invariant if it does not receive any collaborative information from its neighboring agents. Dong et al. [25] presented several sufficient conditions for formation tracking of second-order swarm systems, where each agent was described as a second-order integrator. In this case, the velocity-similar term converges to a constant and the position-similar term linearly diverges away if the convergence constant of the velocity-similar term is nonzero. In [26], formation tracking criteria for high-order linear time-invariant swarm systems were given, where the formation tracking problem is more challenging since the dynamics of each agent is of high order.

For practical swarm systems, the whole energy supply is usually limited and the above literature studies on formation control did not considered the impacts of the limited energy on formation design and analysis problems. Similar to the guaranteed-cost control of isolated systems, the whole guaranteed-cost function was constructed on the basis of the integral principle in $[27,28]$, where sufficient conditions for the guaranteed-cost formation were proposed via the linear matrix inequality tool and the nonzero eigenvalues of the Laplacian matrix of the communication topology. Yu et al. [29] presented the guaranteed-cost time-varying formation criteria for swarm systems with external disturbances and time-varying delays, but they did not give an approach to determine the upper bound of the performance function. Especially, it should be pointed out that the whole energy supply of a swarm system was assumed to be infinite when distributed formation control was carried out in [27-29]. Actually, the whole energy supply is usually limited for practical swarm systems and its impacts on time-varying formation control are critically important. To the best of our knowledge, the design and analysis problems of time-varying formation tracking for high-order linear time-variant swarm systems with the limited energy and fixed topologies are still open and are not comprehensively discussed.
The current paper investigated time-varying formation tracking problems for high-order linear time-variant swarm systems with the limited energy and fixed topologies. Based on the state errors and the formation function errors among neighboring agents including the collaborative information of the leader, a new formation tracking protocol is presented, where an energy integral term is introduced to guarantee that the practical energy consumption is less than or equal to the whole energy supply. Especially, the dynamics of each agent is described by a high-order linear time-invariant model and the communication topology is depicted as a directed graph with a spanning tree. Furthermore, the whole dynamics of the leader-following swarm system is separated as the dynamics of the leader and the relative dynamics among all agents by constructing the specific nonsingular transformation matrix, and design and analysis criteria for time-varying formation tracking with the limited energy are proposed, respectively, where the relationship matrix between the matrix variable and the limited energy is associated with the Laplacian matrix of a star graph with the leader being the central vertex. Moreover, the time-varying formation tracking criteria are extended into the time-invariant ones by simplifying the different formation feasible condition.

The rest part of the current paper is arranged as follows. The communication topology of a leader-following swarm system is depicted and the problem description of formation tracking for a swarm system with the limited energy is revealed in Section 2. Sufficient conditions for formation tracking design and analysis are proposed in Section 3. In Section 4, two numerical examples are illustrated to demonstrate time-varying and time-invariant formation criteria, respectively. Finally, Section 5 summarizes the key features of our conclusions.

1.1. Notations. The symbols $R^{n}$ and $R^{n \times n}$ denote the $n$-dimensional real column vector and the $n$-dimensional real matrix space, respectively. The symbol $\mathbf{1}_{N}$ represents the $N$ dimensional column vector with all components 1 . The notation $\mathbf{0}$ denotes the zero vector or zero matrix with compatible dimensions, and the matrix $I_{r}$ means an identity matrix with dimension $r$. The symbol $\otimes$ represents the Kronecker product. The notation $Q^{T}=Q>0$ shows that the matrix $Q$ is symmetric and positive definite.

\section{Problem Description}

2.1. Modeling Communication Topology. For a swarm system with $N$ homogenous agents and a leader-following communication structure, the fixed topology can be described by a weighed directed graph $G=(V(G), E(G))$, where $V(G)=$ $\left\{v_{0}, v_{1}, \ldots, v_{N}\right\}$ stands for the vertex set with the element $v_{k}(k \in\{0,1, \ldots, N\})$ representing agent $k$ and $E(G)=\left\{e_{k i}=\left(v_{k}, v_{i}\right)\right\}$ denotes the edge set with the element $e_{k i}$ denoting the communication channel between agent $k$ and agent $i$. Without loss of generality, agent 0 is set as the leader and agent $k(k=1,2, \ldots, N)$ are followers. Furthermore, it is assumed that the communication channels 
from the leader to the followers are directional and the communication channels among followers are bidirectional, which means that the leader does not impacted by the followers and only some followers can obtain the collaborative information of the leader.

The symbol $N_{k}=\left\{i:\left(v_{i}, v_{k}\right) \in E(G)\right\}$ is used to represent the neighboring agent set of agent $k$, where each neighboring agent is called a neighbor. The symbol $w_{i k}$ is applied to represent the communication weight between agents $k$ and $i$, where $w_{i k}=0$ if agents $k$ and $i$ are not connected, $w_{i k}>0$ if agents $k$ and $i$ are connected. Especially, it is assumed that there does not exist self-loop; that is, $w_{k k}=$ $0(k=0,1, \ldots, N)$. The matrix $L=\left[l_{i k}\right] \in R^{(N+1) \times(N+1)}$ is applied to denote the Laplacian matrix of the communication topology, where $l_{k k}=\sum_{i \in N_{k}} w_{k i}$ and $l_{i k}=-w_{i k}(k \neq i)$. The row sum of the Laplacian matrix is equal to zero; that is, $L \mathbf{1}_{N}=\mathbf{0}$. Moreover, it is supposed that the fixed communication topology has a spanning tree. In this case, zero is the simple eigenvalue of the Laplacian matrix and all the other eigenvalues are positive. One can find more basic concepts and conclusions on algebraic graph theory in [30].
2.2. Modeling Formation Control. The dynamics of each agent is modeled as the following high-order linear timeinvariant system:

$$
\left\{\begin{array}{l}
\dot{x}_{0}(t)=A x_{0}(t), \\
\dot{x}_{k}(t)=A x_{k}(t)+B u_{k}(t),
\end{array}\right.
$$

where $k=1,2, \ldots, N, A \in R^{n \times n}, B \in R^{n \times m}, x_{0}(t)$ is the collaborative state of the leader, and $x_{k}(t)$ and $u_{k}(t)$ represent the collaborative state and the control input, respectively. Furthermore, a vector-valued function $\eta(t)=\left[\eta_{1}^{T}(t), \eta_{2}^{T}(t), \ldots, \eta_{N}^{T}(t)\right]^{T}$ is applied to design a specific geometric structure for the followers to achieve and maintain, where the element $\eta_{k}(t)$ is the formation function of agent $k(k \in\{1,2, \ldots, N\})$, which has the piecewise continuous differentiable property. The formation structure is time-varying if the vector-valued function $\eta(t)$ is timevarying, and the formation structure is fixed if the derivative of the vector-valued function $\eta(t)$ is zero.

In the sequel, a new formation tracking protocol with an energy integral term and a fixed communication topology is presented as

$$
\left\{\begin{array}{l}
u_{k}(t)=u_{k 0}(t)+u_{k f}(t) \\
u_{k 0}(t)=w_{k 0} K\left(x_{0}(t)-x_{k}(t)+\eta_{k}(t)\right) \\
u_{k f}(t)=K \sum_{i \in N_{k}} w_{k i}\left(x_{i}(t)-\eta_{i}(t)-x_{k}(t)+\eta_{k}(t)\right) \\
J_{e}=\sum_{k=1}^{N} \int_{0}^{+\infty} u_{k}^{T}(t) Q u_{k}(t) \mathrm{d} t
\end{array}\right.
$$

where $k=1,2, \ldots, N, K \in R^{m \times n}, Q^{T}=Q>0$, and $J_{e}$ denotes the practical energy consumption of swarm system (1) as a whole. Actually, the control input $u_{k}(t)$ contains two components $u_{k 0}(t)$ and $u_{k f}(t)$, where the component $u_{k 0}(t)$ denotes the impacts of the leader on the control input of agent $k$ and the component $u_{k f}(t)$ represents the impacts of neighboring followers on the control input of agent $k$.

Let $J_{\max }$ be the energy supply of swarm system (1) as a whole; that is, the energy supply is limited. Now, we, respectively, give the analysis and design definitions of the limited-energy formation tracking of swarm system (1) with tracking protocol (2) as follows.

Definition 1. For any given $J_{\max }>0$ and the control gain $K$, swarm system (1) with tracking protocol (2) is said to be limited-energy formation tracking achievable if $\lim _{t \longrightarrow+\infty}\left(x_{k}(t)-\eta_{k}(t)-x_{0}(t)\right)=\mathbf{0} \quad(k=1,2, \ldots, N)$ and $J_{e} \leq J_{\max }$ for any bounded disagreement initial conditions $x_{k}(0)-\eta_{k}(0)(k=1,2, \ldots, N)$.

Definition 2. For any given $J_{\max }>0$, swarm system (1) is said to be limited-energy formation tracking achievable by tracking protocol (2) if there exists a gain matrix $K$ such that $\lim _{t \longrightarrow+\infty}\left(x_{k}(t)-\eta_{k}(t)-x_{0}(t)\right)=\mathbf{0}(k=1,2, \ldots, N)$ and
$J_{e} \leq J_{\max }$ for any bounded disagreement initial conditions $x_{k}(0)-\eta_{k}(0)(k=1,2, \ldots \infty, N)$.

The key objective of the current paper is to give design and analysis criteria for limited-energy formation tracking of swarm system (1) with tracking protocol (2), where both time-varying formation and time-invariant formation are considered.

Remark 1. Tracking protocol (2) has three new properties. The first one is that tracking protocol (2) has an energy integral term associated with all following agents to guarantee that the practical energy consumption is less than or equal to the limited energy. In this case, it is the key challenge to introduce the limited energy into the dynamics of the whole system and to determine the constrained relationship between the energy supply and the control gain. The second one is that the impacts of the leader and neighboring followers are given, respectively, which can be used to decompose the collaborative state of the leader from the whole dynamics of swarm system (1) with tracking protocol (2). The third one is that the formation functions of neighboring agents are involved in tracking protocol (2), which can determine arbitrary piecewise continuous differentiable structures among followers to track the collaborative state of 
the leader, but it should be pointed out that the formation feasibility is dependent on the dynamics of each agent.

\section{Main Results}

By the linear matrix inequality, this section, respectively, presents sufficient conditions for limited-energy formation tracking design and analysis of swarm system (1) with tracking protocol (2) and time-varying geometric structures. Then, limited-energy time-varying formation tracking criteria are extended into time-invariant formation tracking cases with limited energy.

Let $\zeta_{0}(t)=x_{0}(t)-\eta_{0}(t) \quad$ with $\quad \eta_{0}(t) \equiv \mathbf{0} \quad$ and $\zeta_{k}(t)=x_{k}(t)-\eta_{k}(t), \quad(k=1,2, \ldots, N)$, then one can obtain by tracking protocol (2) that

$$
u_{k}(t)=u_{k 0}(t)+u_{k f}(t)
$$

where $k=1,2, \ldots, N$, and

$$
\begin{aligned}
& u_{k 0}(t)=w_{k 0} K\left(\zeta_{0}(t)-\zeta_{k}(t)\right), \\
& u_{k f}(t)=K \sum_{i \in N_{k}} w_{k i}\left(\zeta_{i}(t)-\zeta_{k}(t)\right) .
\end{aligned}
$$

Thus, the dynamics of follower $k$ can be rewritten as

$$
\dot{\zeta}_{k}(t)=A\left(\zeta_{k}(t)+\eta_{k}(t)\right)+B u_{k}(t)-\dot{\eta}_{k}(t), \quad(k=1,2, \ldots, N) .
$$

Let $\quad \zeta(t)=\left[\zeta_{0}^{T}(t), \zeta_{1}^{T}(t), \ldots, \zeta_{N}^{T}(t)\right]^{T} \quad$ and $\tilde{\eta}(t)=\left[\eta_{0}^{T}(t), \eta_{1}^{T}(t), \ldots, \eta_{N}^{T}(t)\right]^{T}$, and $L_{f f}$ denotes the Laplacian matrix of the communication topology among followers; then, one can find by (3) and (5) that

$$
\dot{\zeta}(t)=\left(I_{N+1} \otimes A-L \otimes B K\right) \zeta(t)+\left(I_{N+1} \otimes A\right) \widetilde{\eta}(t)-\dot{\vec{\eta}}(t),
$$

where

$$
\begin{aligned}
L & =\left[\begin{array}{cc}
0 & 0 \\
-l_{f l} & L_{f f}+\Lambda_{f l}
\end{array}\right], \\
l_{f l} & =\left[w_{10}, w_{20}, \ldots, w_{N 0}\right]^{T}, \\
\Lambda_{f l} & =\operatorname{diag}\left\{w_{10}, w_{20}, \ldots, w_{N 0}\right\} .
\end{aligned}
$$

Let $0<\lambda_{1} \leq \lambda_{2} \leq \cdots \leq \lambda_{N}$ denote nonzero eigenvalues of the Laplacian matrix $L$, which are also the eigenvalues of the matrix $L_{f f}+\Lambda_{f l}$ by the structure of the Laplacian matrix $L$. By the linear matrix inequality technique, the following theorem presents a sufficient condition of limited-energy formation tracking design; that is, a design method of the gain matrix $K$ is proposed to make swarm system (1) with tracking protocol (2) and a fixed communication topology achieve limited-energy formation tracking.

Theorem 1. For any given $J_{\max }>0$, if $A \eta_{k}(t)=\dot{\eta}_{k}(t),(k=$ $1,2, \ldots, N)$ and there exists $\widehat{P}^{T}=\widehat{P}>0$ such that

$$
\begin{gathered}
\widehat{P} A^{T}+A \widehat{P}-2 B B^{T}+\lambda_{1}^{-2} \lambda_{N}^{2} B Q B^{T}<0, \\
(x(0)-\eta(0))^{T}\left(\left[\begin{array}{cc}
N & -\mathbf{1}_{N}^{T} \\
-\mathbf{1}_{N} & I_{N}
\end{array}\right] \otimes I_{n}\right)(x(0)-\eta(0)) I_{n} \leq J_{\max } \widehat{P},
\end{gathered}
$$

then swarm system (1) is limited-energy time-varying formation tracking achievable by tracking protocol (2) with $K=\lambda_{1}^{-1} B^{T} \widehat{P}$.

Proof of Theorem 1. A nonsingular matrix is introduced as follows:

$$
W=\left[\begin{array}{cc}
1 & \mathbf{0} \\
\mathbf{1}_{N} & I_{N}
\end{array}\right] .
$$

Whose invertible matrix is

$$
W^{-1}=\left[\begin{array}{cc}
1 & \mathbf{0} \\
-\mathbf{1}_{N} & I_{N}
\end{array}\right] .
$$

It can be shown that $\Lambda_{f f} \mathbf{1}_{N}=l_{f l}$, so one can deduce that

$$
\begin{aligned}
W^{-1} L W & =\left[\begin{array}{ll}
0 & \\
& L_{f f}+\Lambda_{f l}
\end{array}\right], \\
\left(W^{-1} \otimes I_{n}\right) \zeta(t) & =\left[x_{0}^{T}(t), \widetilde{\zeta}_{1}^{T}(t), \ldots, \widetilde{\zeta}_{N}^{T}(t)\right]^{T},
\end{aligned}
$$

where $\widetilde{\zeta}_{k}(t)=\zeta_{k}(t)-x_{0}(t) \quad(k=1,2, \ldots, N)$. Because the communication channels among followers are bidirectional, the Laplacian matrix $L_{f f}$ is symmetric. Since it is assumed that the whole communication topology has a spanning tree, the matrix $\Lambda_{f l}$ is nonzero. In this case, the matrix $L_{f f}+\Lambda_{f l}$ is symmetric and positive definite. Thus, one can find an orthonormal matrix $\widehat{W} \in R^{N \times N}$ such that $\widehat{W}^{T}\left(L_{f f}+\Lambda_{f l}\right) \widehat{W}=\operatorname{diag}\left\{\lambda_{1}, \lambda_{2}, \ldots, \lambda_{N}\right\}$, where $\lambda_{k}>0,(k=$ $1,2, \ldots, N)$ are nonzero eigenvalues of the Laplacian matrix $L$ of the whole communication topology. Let $\tilde{\zeta}(t)=\left[\tilde{\zeta}_{1}^{T}(t), \tilde{\zeta}_{2}^{T}(t), \ldots, \tilde{\zeta}_{N}^{T}(t)\right]^{T} \quad$ and $\quad \widehat{\zeta}(t)=\left(\widehat{W}^{T} \otimes\right.$ $\left.I_{n}\right) \widetilde{\zeta}(t)=\left[\widehat{\zeta}_{1}^{T}(t), \widehat{\zeta}_{2}^{T}(t), \ldots, \widehat{\zeta}_{N}^{T}(t)\right]^{T}$, then swarm system $(1)$ with tracking protocol (2) can be transformed by (6) into

$$
\begin{aligned}
& \dot{x}_{0}(t)=A x_{0}(t), \\
& \dot{\widehat{\zeta}}_{k}(t)=\left(A-\lambda_{k} B K\right) \widehat{\zeta}_{k}(t)+\left(e_{k}^{T} \widehat{W}^{T}\left[0, I_{N}\right] W^{-1} \otimes A\right) \widetilde{\eta}(t)-\left(e_{k}^{T} \widehat{W}^{T}\left[0, I_{N}\right] W^{-1} \otimes I_{n}\right) \dot{\tilde{\eta}}(t),
\end{aligned}
$$

where $k=1,2, \ldots, N$ and the column vector $e_{k}$ is $N$-dimensional and its $k$-th component is 1 and zero elsewhere. Because $\widetilde{\zeta}_{k}(t)=\zeta_{k}(t)-x_{0}(t) \quad(k=1,2, \ldots, N)$ and $\widehat{W}$ is orthonormal, it can be found that if $\lim _{t \rightarrow+\infty} \widehat{\zeta}_{k}(t)=\mathbf{0} \quad(k=1,2, \ldots, N)$, then one can obtain that 


$$
\lim _{t \longrightarrow \infty}\left(x_{k}(t)-\eta_{k}(t)-x_{0}(t)\right)=0, \quad(k=1,2, \ldots, N),
$$

which means that swarm system (1) with tracking protocol (2) achieves formation tracking.

Furthermore, we give an approach to design $K$ such that $\lim _{t \rightarrow+\infty} \widehat{\zeta}_{k}(t)=\mathbf{0}, \quad(k=1,2, \ldots, N)$. Let $P^{T}=P>0$, then the following Lyapunov function candidate is adopted:

$$
V_{k}(t)=\widehat{\zeta}_{k}^{T}(t) P \widehat{\zeta}_{k}(t), \quad(k=1,2, \ldots, N)
$$

By taking the time derivative of $V_{k}(t)$, it can be derived that

$$
\begin{aligned}
\dot{V}_{k}(t)= & \widehat{\zeta}_{k}^{T}(t)\left(\left(A-\lambda_{k} B K\right)^{T} P+P\left(A-\lambda_{k} B K\right)\right) \widehat{\zeta}_{k}(t), \\
& +2 \widehat{\zeta}_{k}^{T}(t) P\left(e_{k}^{T} \widehat{W}^{T}\left[\mathbf{0}, I_{N}\right] W^{-1} \otimes A\right) \tilde{\eta}(t), \\
& -2 \widehat{\zeta}_{k}^{T}(t) P\left(e_{k}^{T} \widehat{W}^{T}\left[\mathbf{0}, I_{N}\right] W^{-1} \otimes I_{n}\right) \dot{\bar{\eta}}(t) .
\end{aligned}
$$

Let $K=\lambda_{1}^{-1} B^{T} P$, then one has

$$
\left(A-\lambda_{k} B K\right)^{T} P+P\left(A-\lambda_{k} B K\right) \leq A^{T} P+P A-2 \lambda_{1}^{-1} \lambda_{k} P B B^{T} P .
$$

Since the nonzero eigenvalues of the Laplacian matrix $L$ satisfy that $0<\lambda_{1} \leq \lambda_{2} \leq \cdots \leq \lambda_{N}$, one can deduce that $\lambda_{1}^{-1} \lambda_{k} \geq 1(k=1,2, \ldots, N)$. Therefore, it can be derived by (16) that

$$
A^{T} P+P A-2 \lambda_{1}^{-1} \lambda_{k} P B B^{T} P \leq A^{T} P+P A-2 P B B^{T} P .
$$

Due to

$$
\begin{aligned}
e_{k}^{T} \widehat{W}^{T}\left[\mathbf{0}, I_{N}\right] W^{-1} \otimes A & =\left(e_{k}^{T} \widehat{W}^{T}\left[\mathbf{0}, I_{N}\right] W^{-1} \otimes I_{n}\right)\left(I_{N+1} \otimes A\right), \\
A \eta_{k}(t)-\dot{\eta}_{k}(t) & =0, \quad(k=1,2, \ldots, N),
\end{aligned}
$$

one can obtain that

$$
\left(e_{k}^{T} \widehat{W}^{T}\left[\mathbf{0}, I_{N}\right] W^{-1} \otimes A\right) \widetilde{\eta}(t)-\left(e_{k}^{T} \widehat{W}^{T}\left[\mathbf{0}, I_{N}\right] W^{-1} \otimes I_{n}\right) \dot{\bar{\eta}}(t)=\mathbf{0} .
$$

By (15), (17), and (19), if

$$
A^{T} P+P A-2 P B B^{T} P<0,
$$

then one can deduce that

$$
\lim _{t \longrightarrow \infty} \widehat{\zeta}_{k}(t)=0, \quad(k=1,2, \ldots, N) .
$$

That is, swarm system (1) with tracking protocol (2) achieves formation tracking. In the other work, swarm system (1) is formation tracking achievable by tracking protocol (2) with $K=\lambda_{1}^{-1} B^{T} P$.

In the sequel, the impacts of the limited energy are dealt with. One can show that

$$
\sum_{k=1}^{N} u_{k}^{T}(t) Q u_{k}(t)=\widetilde{\zeta}^{T}(t)\left(\left(L_{f f}+\Lambda_{f l}\right)^{2} \otimes K^{T} Q K\right) \tilde{\zeta}(t)
$$

Due to $\widehat{\zeta}(t)=\left(\widehat{W}^{T} \otimes I_{n}\right) \widetilde{\zeta}(t)$, one can deduce that $\widetilde{\zeta}^{T}(t)\left(\left(L_{f f}+\Lambda_{f l}\right)^{2} \otimes K^{T} Q K\right) \widetilde{\zeta}(t)=\sum_{k=1}^{N} \lambda_{k}^{2} \widehat{\zeta}_{k}^{T}(t) K^{T} Q K \widehat{\zeta}_{k}(t)$

Since $K=\lambda_{1}^{-1} B^{T} P$, it can be found by (22) and (23) that $\sum_{k=1}^{N} u_{k}^{T}(t) Q u_{k}(t)=\lambda_{1}^{-2} \sum_{k=1}^{N} \lambda_{k}^{2} \widehat{\zeta}_{k}^{T}(t) P B Q B^{T} P \widehat{\zeta}_{k}(t)$

Let $\kappa \geq 0$, then one can see that

$$
\begin{aligned}
J_{e}^{\kappa} & =\int_{0}^{\kappa} \widetilde{\zeta}^{T}(t)\left(\left(L_{f f}+\Lambda_{f l}\right)^{2} \otimes K^{T} Q K\right) \tilde{\zeta}(t) \mathrm{d} t, \\
& =\lambda_{1}^{-2} \sum_{i=1}^{N} \int_{0}^{\kappa} \lambda_{k}^{2} \hat{\zeta}_{k}^{T}(t) P B Q B^{T} P \hat{\zeta}_{k}(t) \mathrm{d} t .
\end{aligned}
$$

According to $\int_{0}^{\kappa} \dot{V}_{k}(t) \mathrm{d} t=V_{k}(\kappa)-V_{k}(0)$, from (22) to (25), one can find that

$$
\begin{aligned}
J_{e}^{\kappa}= & \sum_{k=1}^{N} \int_{0}^{\kappa}\left(\left(\dot{V}_{k}(t)+\lambda_{1}^{-2} \lambda_{k}^{2} \widehat{\zeta}_{k}^{T}(t) P B Q B^{T} P \widehat{\zeta}_{k}(t)\right) \mathrm{d} t-V_{k}(\kappa)\right) \\
& +\sum_{k=1}^{N} V_{k}(0) .
\end{aligned}
$$

Because $\lambda_{N}$ is the maximum nonzero eigenvalue of the Laplacian matrix $L$ of the whole communication topology, it can be obtained by (26) that

$$
\begin{aligned}
J_{e}^{\kappa} \leq & \left.\sum_{k=1}^{N} \int_{0}^{\kappa}\left(\left(\dot{V}_{k}(t)+\lambda_{1}^{-2} \lambda_{N}^{2} \widehat{\zeta}_{k}^{T}(t) P B Q B^{T} P \widehat{\zeta}_{k}(t)\right)\right) \mathrm{d} t-V_{k}(\kappa)\right) \\
& +\sum_{k=1}^{N} V_{k}(0) \cdot \sqrt{b^{2}-4 a c .}
\end{aligned}
$$

Thus, one can find that if

$$
A^{T} P+P A-2 P B B^{T} P+\lambda_{1}^{-2} \lambda_{N}^{2} P B Q B^{T} P<0,
$$

then one has $\lim _{t \longrightarrow+\infty} \widehat{\zeta}_{k}(t)=\mathbf{0}$ and $\lim _{\kappa \longrightarrow+\infty} V_{k}(\kappa)=\mathbf{0}, \quad(k=1,2, \ldots, N)$. In this case, one can deduce by (27) that

$$
J_{e} \leq \sum_{k=1}^{N} \widehat{\zeta}_{k}^{T}(0) P \widehat{\zeta}_{k}(0)
$$
has

Since $\widehat{\zeta}_{k}(0)=e_{k}^{T}\left(\widehat{W}^{T} \otimes I_{n}\right) \widetilde{\zeta}(0), \quad(k=1,2, \ldots, N)$, one

$$
\sum_{k=1}^{N} \widehat{\zeta}_{k}^{T}(0) P \widehat{\zeta}_{k}(0)=\widetilde{\zeta}^{T}(0)\left(I_{N} \otimes P\right) \widetilde{\zeta}(0)
$$


Due to

$$
\begin{aligned}
\widetilde{\zeta}(0) & =\left(\left[\mathbf{0}, I_{N}\right] W^{-1} \otimes I_{n}\right) \zeta(0), \\
W^{-1} & =\left[\begin{array}{cc}
1 & \mathbf{0} \\
-\mathbf{1}_{N} & I_{N}
\end{array}\right] .
\end{aligned}
$$

It can be found that

$$
\left(\left[\mathbf{0}, I_{N}\right] W^{-1}\right)^{T}\left(\left[\mathbf{0}, I_{N}\right] W^{-1}\right)=\left[\begin{array}{cc}
N & -\mathbf{1}_{N}^{T} \\
-\mathbf{1}_{N} & I_{N}
\end{array}\right] .
$$

Thus, one can find by (30) that

$$
\sum_{k=1}^{N} \widehat{\zeta}_{k}^{T}(0) P \widehat{\zeta}_{k}(0)=\widetilde{\zeta}^{T}(0)\left(\left[\begin{array}{cc}
N & -\mathbf{1}_{N}^{T} \\
-\mathbf{1}_{N} & I_{N}
\end{array}\right] \otimes P\right) \widetilde{\zeta}(0) .
$$

Because it is supposed that $x_{k}(0)-\eta_{k}(0), \quad(k=1,2, \ldots, N)$ are disagreement, there must exist $\widehat{\zeta}_{k}(0), \quad(k \in\{1,2, \ldots, N\})$ is nonzero. In this case, one can show that

$$
\zeta^{T}(0)\left(\left[\begin{array}{cc}
N & -\mathbf{1}_{N}^{T} \\
-\mathbf{1}_{N} & I_{N}
\end{array}\right] \otimes I_{n}\right) \zeta(0)=\sum_{k=1}^{N} \widehat{\zeta}_{k}^{T}(0) \widehat{\zeta}_{k}(0)>0 .
$$

Hence, there exists $\gamma>0$ such that

$$
J_{\max }=\zeta^{T}(0)\left(\left[\begin{array}{cc}
N & -\mathbf{1}_{N}^{T} \\
-\mathbf{1}_{N} & I_{N}
\end{array}\right] \otimes \gamma I_{n}\right) \zeta(0) .
$$

Since the matrix $\left[\begin{array}{cc}N & -\mathbf{1}_{N}^{T} \\ -\mathbf{1}_{N} & I_{N}\end{array}\right]$ has a simple zero eigenvalue and $N-1$ positive eigenvalues, one can find by (33) and (35) that $P \leq \gamma I_{n}$ can ensure that $J_{e} \leq J_{\max }$; that is, it is required that

$$
(x(0)-\eta(0))^{T}\left(\left[\begin{array}{cc}
N & -\mathbf{1}_{N}^{T} \\
-\mathbf{1}_{N} & I_{N}
\end{array}\right] \otimes I_{n}\right)(x(0)-\eta(0)) P \leq J_{\max } I_{n} .
$$

Let $\widehat{P}=P^{-1}$, then the conclusion of Theorem 1 can be deduced

Theorem 1 gives a sufficient condition for limited-energy formation design, which proposes a design method of the gain matrix of tracking protocol (2). For the case that the gain matrix is given, by the Schur lemma in [31] and the convex property of the linear matrix inequality, the following theorem can be obtained directly on the basis of the Proof of Theorem 1, which presents a sufficient condition for limited-energy formation tracking analysis.

Theorem 2. For any given $J_{\max }>0$ and the gain matrix $K$, if $A \eta_{k}(t)=\dot{\eta}_{k}(t), \quad(k=1,2, \ldots, N)$ and there exists $P^{T}=P>0$ such that

$$
\begin{gathered}
{\left[\begin{array}{cc}
A^{T} P+P A-\lambda_{k} P B K-\lambda_{k} K^{T} B^{T} P & \lambda_{N} K^{T} Q \\
\lambda_{N} Q K & -Q
\end{array}\right]<0, \quad(k=1, N),} \\
(x(0)-\eta(0))^{T}\left(\left[\begin{array}{cc}
N & -\mathbf{1}_{N}^{T} \\
-\mathbf{1}_{N} & I_{N}
\end{array}\right] \otimes I_{n}\right)(x(0)-\eta(0)) P \leq J_{\max } I_{n},
\end{gathered}
$$

then swarm system (1) with tracking protocol (2) achieves limited-energy time-varying formation tracking.

Moreover, if the formation structure among followers is time-invariant, then one can obtain the following two conclusions by the above analysis, which present limited-energy formation tracking design and analysis criteria, respectively.

Theorem 3. For any given $J_{\max }>0$, if $A \eta_{k}(t)=\mathbf{0}, \dot{\eta}_{k}(t)=$ 0, $\quad(k=1,2, \ldots, N)$ and there exists $\widehat{P}^{T}=\widehat{P}>0$ such that

$$
\begin{gathered}
\widehat{P} A^{T}+A \widehat{P}-2 B B^{T}+\lambda_{1}^{-2} \lambda_{N}^{2} B Q B^{T}<0, \\
(x(0)-\eta(0))^{T}\left(\left[\begin{array}{cc}
N & -\mathbf{1}_{N}^{T} \\
-\mathbf{1}_{N} & I_{N}
\end{array}\right] \otimes I_{n}\right)(x(0)-\eta(0)) I_{n} \leq J_{\max } \widehat{P},
\end{gathered}
$$

then swarm system (1) is limited-energy time-invariant formation tracking achievable by tracking protocol (2) with $K=\lambda_{1}^{-1} B^{T} \widehat{P}^{-1}$.

Theorem 4. For any given $J_{\max }>0$, the gain matrix $K$, if $A \eta_{k}(t)=\mathbf{0}, \dot{\eta}_{k}(t)=\mathbf{0}, \quad(k=1,2, \ldots, N)$ and there exists $P^{T}=P>0$ such that

$$
\begin{gathered}
{\left[\begin{array}{cc}
A^{T} P+P A-\lambda_{k} P B K-\lambda_{k} K^{T} B^{T} P & \lambda_{N} K^{T} Q \\
\lambda_{N} Q K & -Q
\end{array}\right]<0, \quad(k=1, N),} \\
(x(0)-\eta(0))^{T}\left(\left[\begin{array}{cc}
N & -\mathbf{1}_{N}^{T} \\
-\mathbf{1}_{N} & I_{N}
\end{array}\right] \otimes I_{n}\right)(x(0)-\eta(0)) P \leq J_{\max } I_{n},
\end{gathered}
$$


then swarm system (1) with tracking protocol (2) achieves limited-energy time-invariant formation tracking.

Remark 2. By choosing different formation functions with the piecewise continuous differentiable property, the different formation structures can be achieved for followers in swarm systems with leader-following structures to maintain. From Theorems 1 to 4, it can be found that some formation structures may be unfeasible, and the feasible property depends on the dynamics of each follower; that is, the feasible condition $A \eta_{k}(t)=\dot{\eta}_{k}(t), \quad(k=1,2, \ldots, N)$ must be satisfied for time-varying formation cases and the feasible conditions $A \eta_{k}(t)=\mathbf{0}$ and $\dot{\eta}_{k}(t)=\mathbf{0}, \quad(k=1,2, \ldots, N)$ should hold for time-invariant formation cases. Furthermore, the relationship matrix $\left[\begin{array}{cc}N & -\mathbf{1}_{N}^{T} \\ -\mathbf{1}_{N} & I_{N}\end{array}\right]$ between the limited energy and the matrix variable essentially is the Laplacian matrix of a star graph with all edge weights one, where the leader is the central vertex and followers are not connected with each other. This is coincident with the leader-following communication structure of the whole swarm system. Especially, sufficient conditions in Theorems 1 to 4 include the minimum and maximum nonzero eigenvalues of the Laplacian matrix, whose precise values are difficult to be solved, but they can be estimated by the proposed methods in $[32,33]$, respectively. It should be pointed out that sufficient conditions in Theorems 1 to 4 can be checked by the FEASP solver of the Matlab's LMI toolbox in [34].

\section{Numerical Examples}

This section presents two numerical examples to demonstrate the effectiveness of main conclusions about limited- energy formation tracking for swarm systems with fixed communication topologies.

Example 1 (time-varying formation tracking). Consider a swarm system with six agents and a leader-following communication structure, where the dynamics of each agent is described by (1) with

$$
\begin{aligned}
& A=\left[\begin{array}{ccc}
0 & 1 & 2 \\
3 & 2 & 3 \\
-2 & -2 & -3
\end{array}\right], \\
& B=\left[\begin{array}{cc}
1 & -1 \\
-1 & -1 \\
0 & 0
\end{array}\right] .
\end{aligned}
$$

The communication topology with a leader-following structure is revealed by Figure 1, where agent 0 is the leader, agents 1 to 5 are followers, and the edge weight is set to be 0 1 ; that is, the weight of the connected edge is one and the weight of the unconnected edge is zero. The initial states of this swarm system are given as follows:

$$
\begin{aligned}
& x_{0}(0)=[1.50,0.40,-3.20]^{T}, \\
& x_{1}(0)=[-1.51,1.48,-6.85]^{T}, \\
& x_{2}(0)=[6.15,0.73,7.92]^{T}, \\
& x_{3}(0)=[5.99,6.66,2.62]^{T}, \\
& x_{4}(0)=[-4.38,-4.23,-0.37]^{T}, \\
& x_{5}(0)=[2.04,-3.27,2.51]^{T} .
\end{aligned}
$$
by

The formation structure among followers is determined

$$
\eta_{k}(t)=\left[\begin{array}{c}
\sin \left(t+\frac{2(k-1) \pi}{5}\right)-\cos \left(t+\frac{2(k-1) \pi}{5}\right) \\
-3 \sin \left(t+\frac{2(k-1) \pi}{5}\right)-3 \cos \left(t+\frac{2(k-1) \pi}{5}\right) \\
2 \sin \left(t+\frac{2(k-1) \pi}{5}\right)+2 \cos \left(t+\frac{2(k-1) \pi}{5}\right)
\end{array}\right], \quad(k=1,2, \ldots, 5) .
$$

It is clear that the formation functions satisfy that $A \eta_{k}(t)=\dot{\eta}_{k}(t), \quad(k=1,2, \ldots, 5)$ in Theorem 1. Let $Q=$ $0.1 I_{2}$ and $J_{\max }=40$. By the FEASP solver, one can obtain by Theorem 1 that

$$
P=\left[\begin{array}{lll}
285.68 & 299.00 & 394.00 \\
299.00 & 357.38 & 434.39 \\
394.00 & 434.39 & 597.76
\end{array}\right]
$$

In this case, one can obtain that

$$
K=\left[\begin{array}{ccc}
-99.44 & -435.72 & -301.45 \\
-4364.10 & -4899.26 & -6183.20
\end{array}\right]
$$

Figure 2 shows the trajectories of $\varsigma_{k}(t), \quad(k=1,2, \ldots, 5)$, where the curves formed by circle markers are the trajectories of the collaborative state of the leader.

In Figure 3, the state snapshots of the leader and five followers are shown at $t=0 \mathrm{~s}, t=16 \mathrm{~s}, t=18 \mathrm{~s}$, and $t=20 \mathrm{~s}$, where the leader is depicted by pentagrams and five 


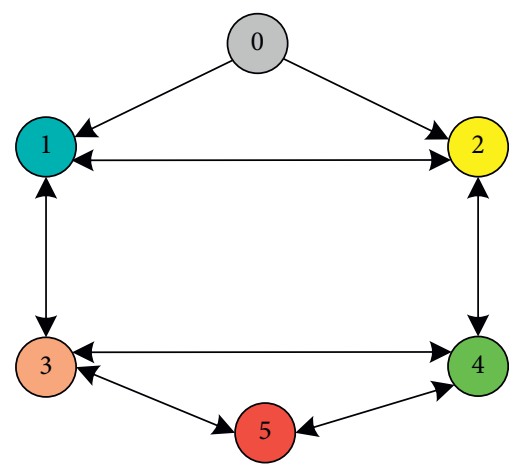

Figure 1: Communication topology for time-varying formation tracking case.

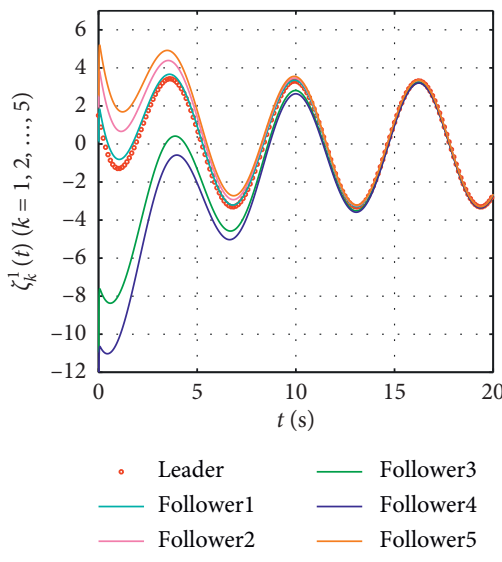

(a)

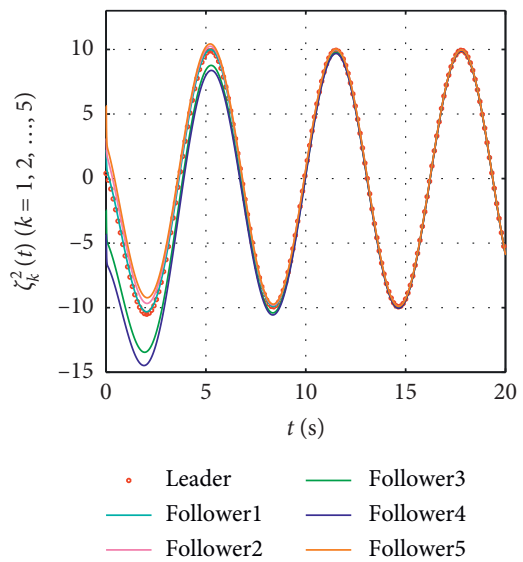

(b)

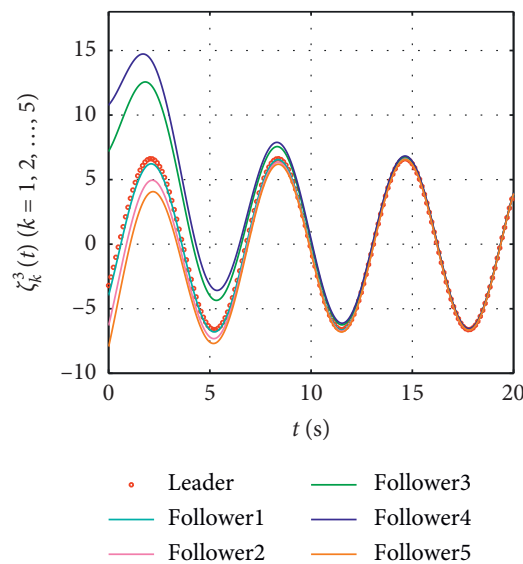

(c)

Figure 2: Trajectories of $\varsigma_{k}(t),(k=1,2, \ldots, 5)$ for time-varying formation tracking case.

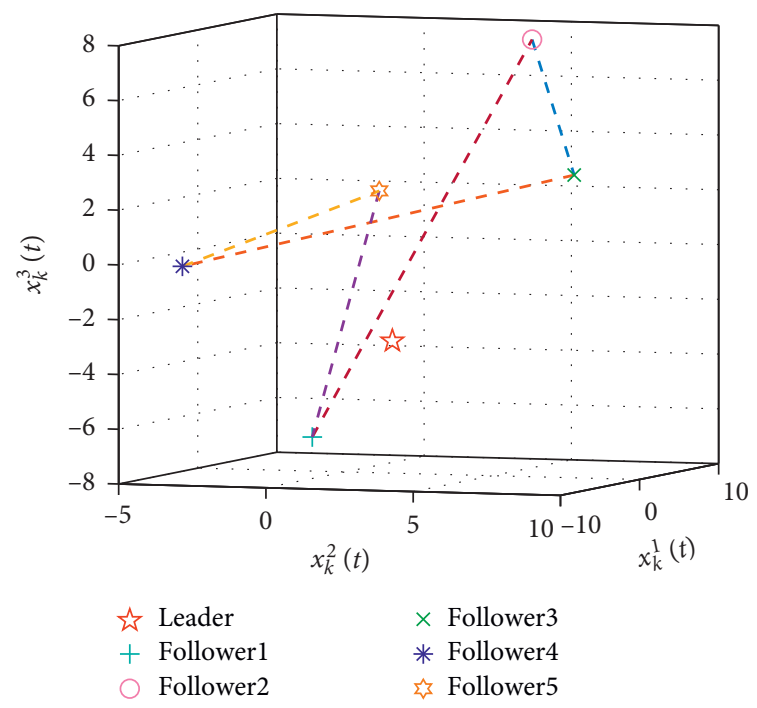

(a)

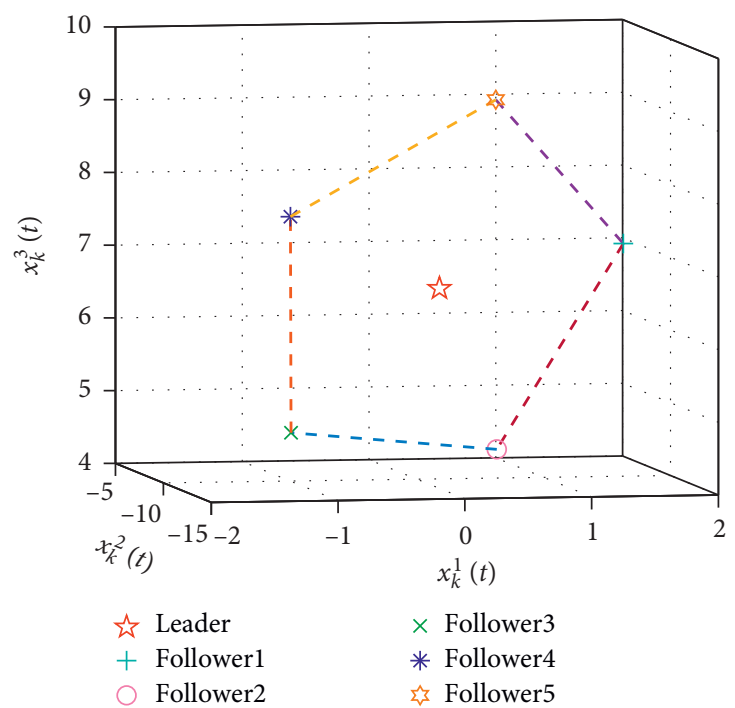

(b)

Figure 3: Continued. 


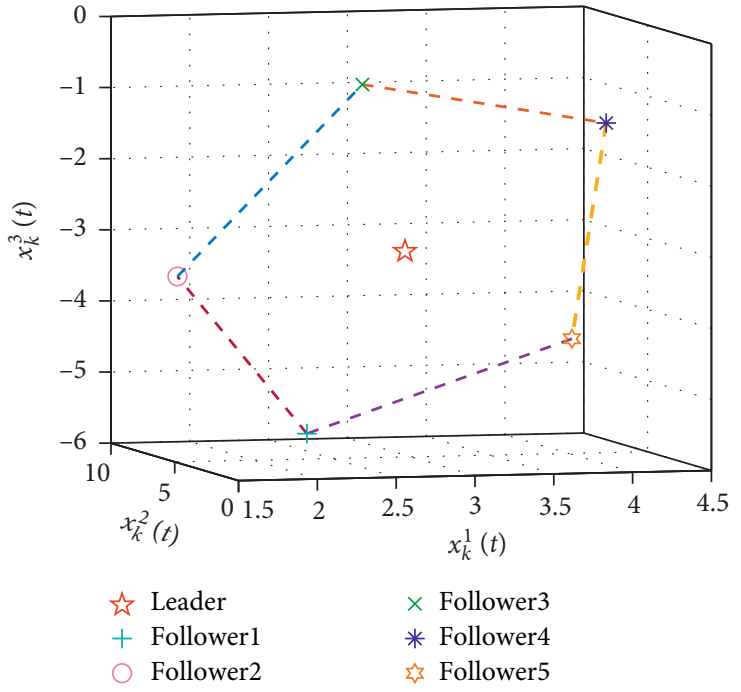

(c)

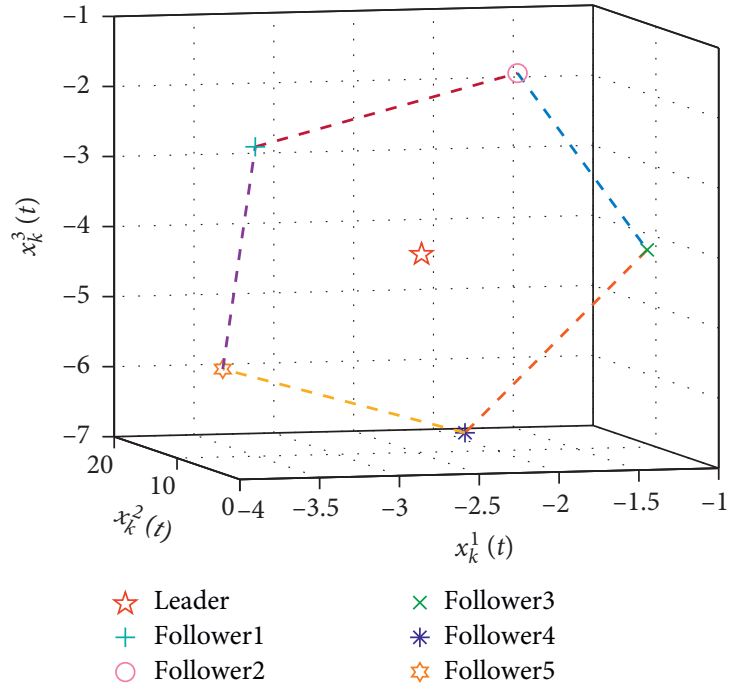

(d)

Figure 3: State snapshots of all the agents at different time for time-varying formation tracking case: (a) $t=0 \mathrm{~s}$, (b) $t=16 \mathrm{~s}$, (c) $t=18 \mathrm{~s}$, and (d) $t=20 \mathrm{~s}$.

followers are described by plusses, circles, $\mathrm{x}$-marks, asterisks, and hexagrams, respectively, where it can be seen that five followers achieve a time-varying pentagon to track the collaborative state of the leader. Figure 4 reveals that the leader counterclockwise rotates about 3.18 cycles, where the initial state is represented by a blue pentagram and the final state is depicted by a black pentagram. In Figure 5, it is shown that the practical energy consumption $J_{e}(t)$ converges to a finite value less than $J_{\max }$. From Figures 2 to 5 , it can be found that this swam system achieves limited-energy time-varying formation tracking.

Example 2 (time-invariant formation tracking). In this example, the leader-following swarm system consists of one leader and eight followers, whose communication topology is shown in Figure 6. In Figure 6, agent 0 is identified as the leader and agents $1-8$ as followers, and without loss of generality, the edge weight among the nine agents is also set as $0-1$.

The matrix pair $(A, B)$ of the dynamics equation of each follower is given as

$$
\begin{aligned}
& A=\left[\begin{array}{ccc}
0 & 1 & -1 \\
0 & 0 & 0 \\
0 & 0 & 0
\end{array}\right], \\
& B=\left[\begin{array}{ll}
0 & 0 \\
0 & 1 \\
1 & 0
\end{array}\right],
\end{aligned}
$$

where $A$ is the system matrix of the leader. The initial values of the states for the leader are given as $x_{0}(0)=[3.5,-2,2]^{T}$, and eight followers are initialized as

$$
\begin{aligned}
& x_{1}(0)=[12.04,5.41,-4.98]^{T}, \\
& x_{2}(0)=[-8.11,4.72,3.04]^{T}, \\
& x_{3}(0)=[-5.64,-3.04,-2.63]^{T}, \\
& x_{4}(0)=[6.73,-4.68,5.52]^{T}, \\
& x_{5}(0)=[-10.98,-2.22,-3.63]^{T}, \\
& x_{6}(0)=[-11.21,-1.17,2.40]^{T}, \\
& x_{7}(0)=[2.95,3.97,-4.60]^{T}, \\
& x_{8}(0)=[9.56,2.60,5.38]^{T} .
\end{aligned}
$$

The purpose of this example is to make eight followers track the leader with a time-invariant formation, which is described by a regular octagon geometric structure. The corresponding time-invariant formation function is described by

$$
\eta_{k}(t)=\left[\begin{array}{c}
\sqrt{2} \cos \left(\frac{(k-1) \pi}{4}\right) \\
\sin \left(\frac{(k-1) \pi}{4}\right) \\
\sin \left(\frac{(k-1) \pi}{4}\right)
\end{array}\right], \quad(k=1,2, \ldots, 8) .
$$

One can find that the formation function $\eta_{k}(t)$ satisfies the condition that $A \eta_{k}(t)=\mathbf{0}$ and $\dot{\eta}_{k}(t)=0, \quad(k=1,2, \ldots, 8)$ in Theorem 2. Let $Q=0.12 I_{2}$ and $J_{\max }=300$; then, by using the FEASP solver, it can be found by Theorem 2 that 


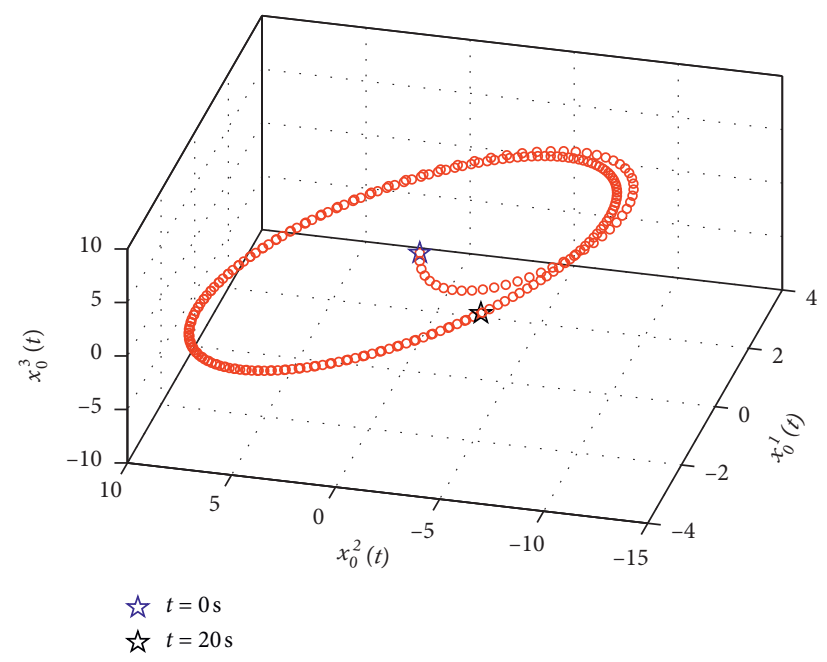

FIgURE 4: Movement track of the leader for time-varying formation tracking case.

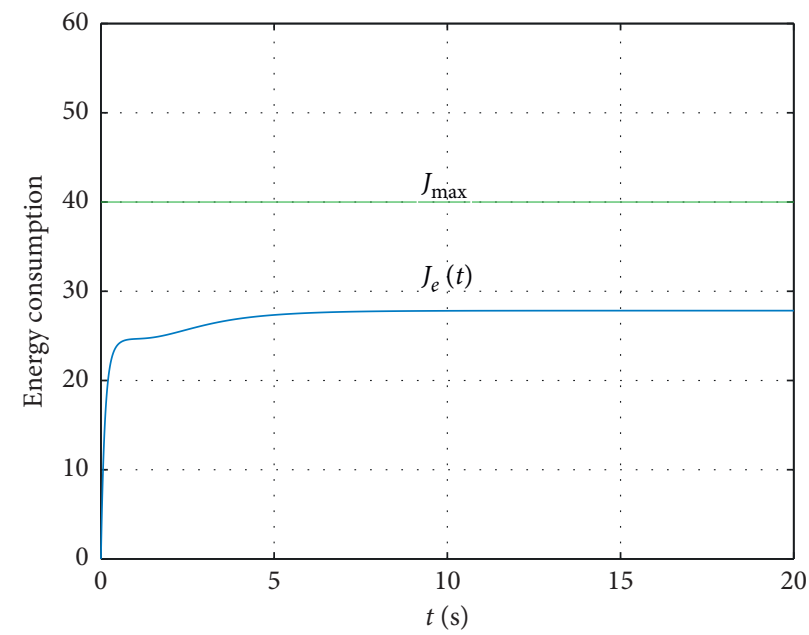

FIGURE 5: Energy consumption and the limited budget for time-varying formation tracking case.

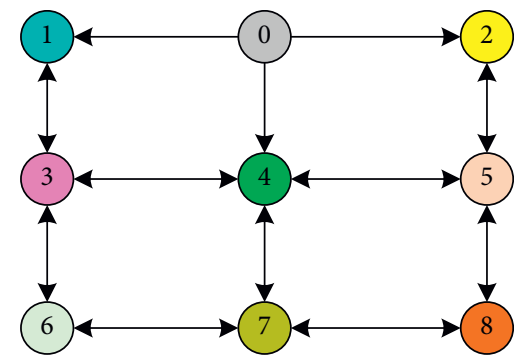

FIGURE 6: Communication topology for time-invariant formation tracking case.

$$
P=\left[\begin{array}{ccc}
4.0614 & 4.0463 & -4.0463 \\
4.0463 & 69.3470 & -8.0918 \\
-4.0463 & -8.0918 & 69.3470
\end{array}\right] \text {. }
$$

In this case, it can be deduced that

$$
K=\left[\begin{array}{ccc}
-1.5794 & -3.1584 & 27.0677 \\
1.5794 & 27.0677 & -3.1584
\end{array}\right] \text {. }
$$

In Figure 7 , the trajectories of $\varsigma_{k}(t),(k=1,2, \ldots, 8)$ are depicted, where the curves formed by circle markers 


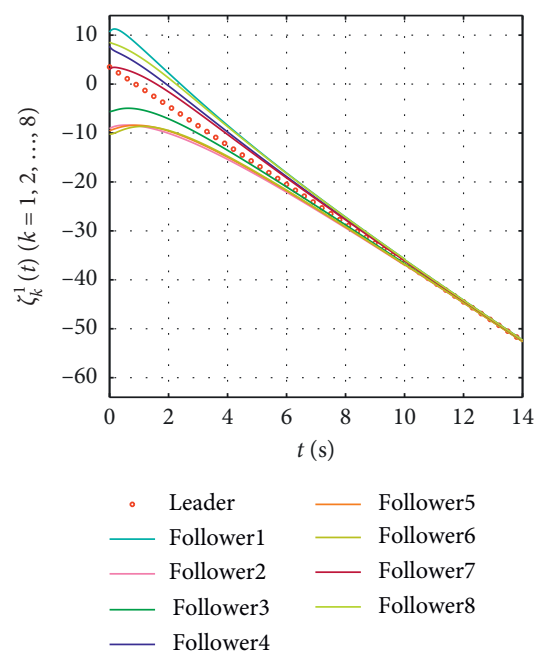

(a)

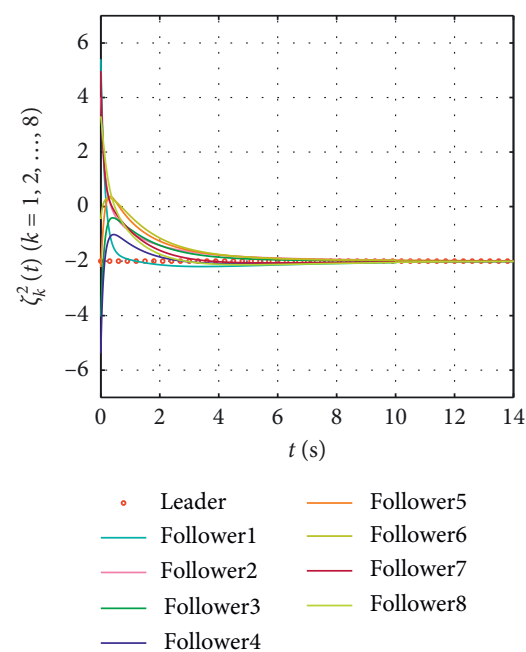

(b)

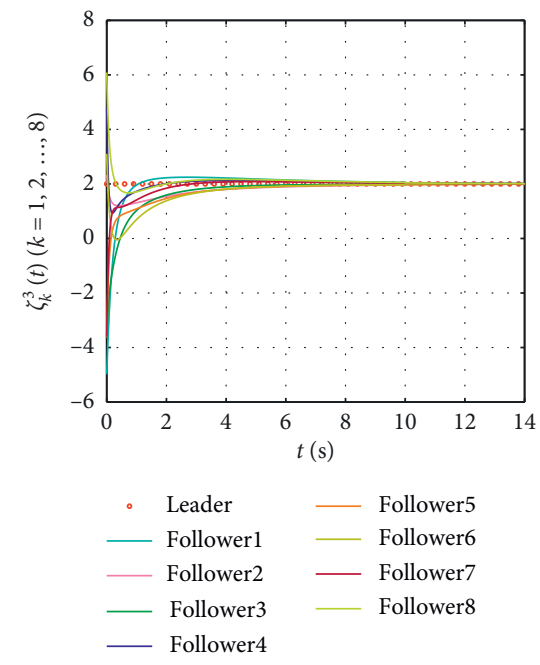

(c)

FIgURE 7: Trajectories of $\varsigma_{k}(t),(k=1,2, \ldots, 8)$ for time-invariant formation tracking case.

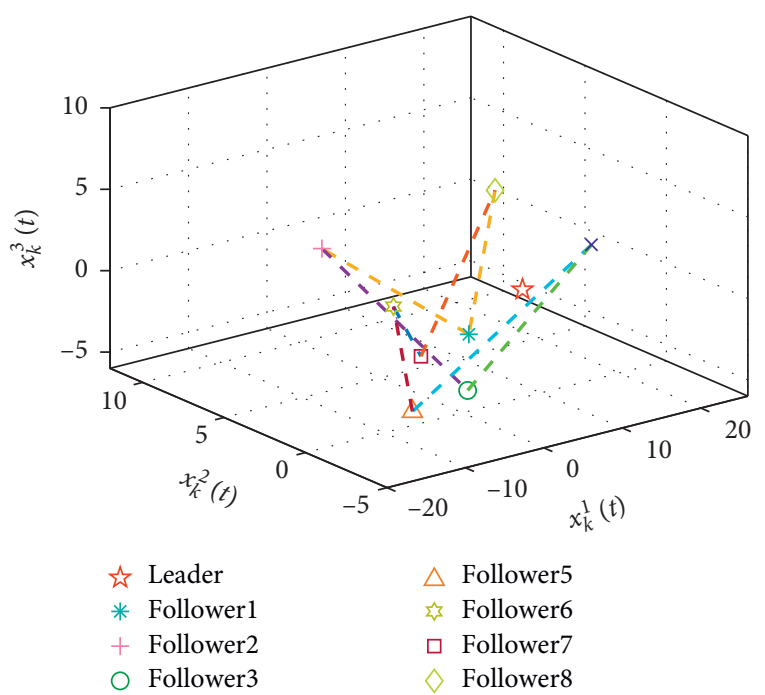

(a)

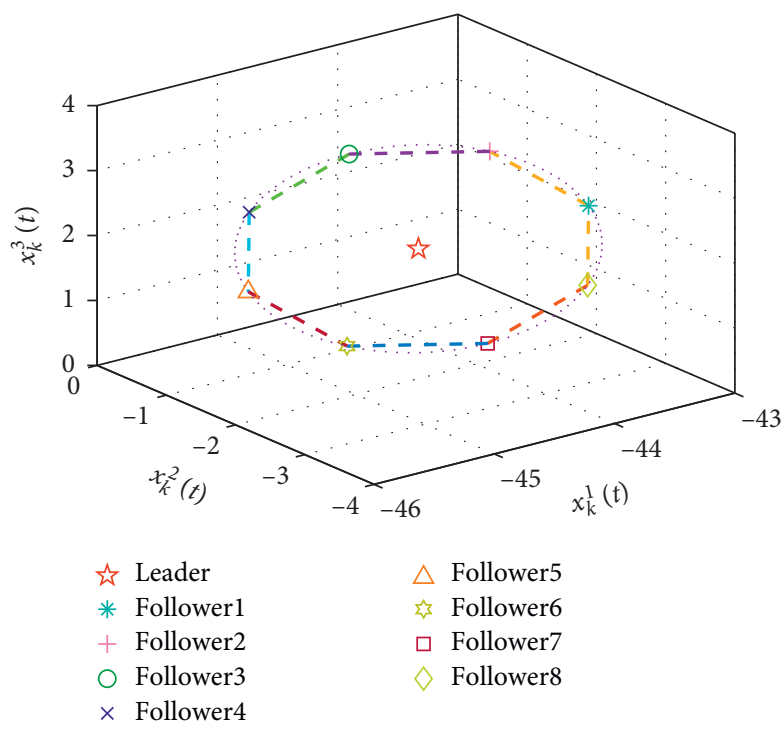

(b)

Figure 8: Continued. 


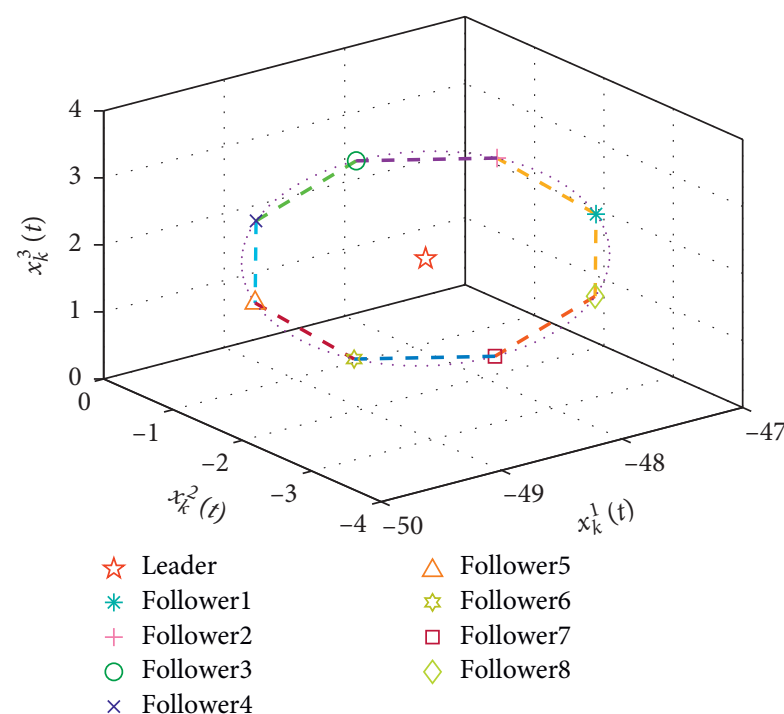

(c)

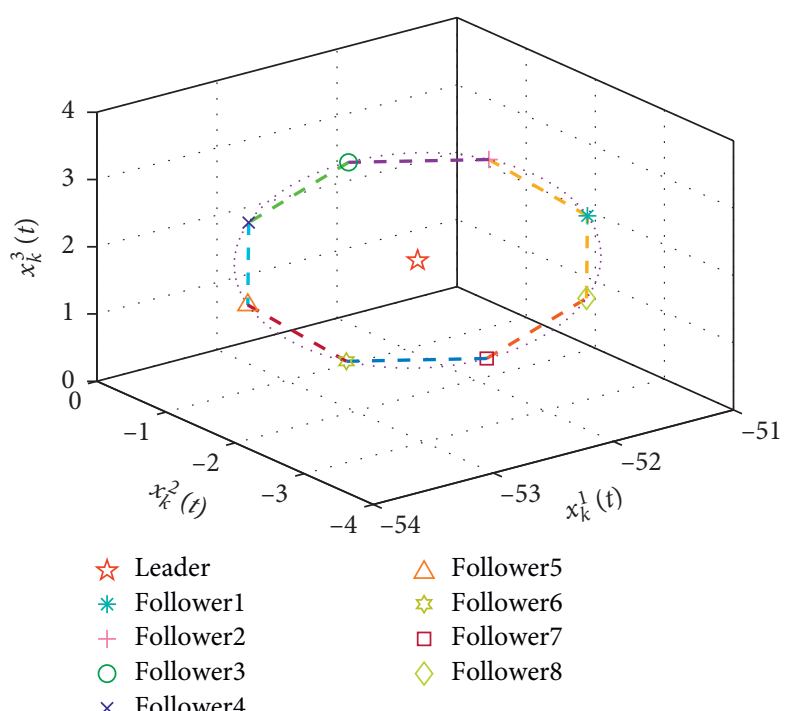

(d)

Figure 8: State snapshots of all the agents at different time for time-invariant formation tracking case: (a) $t=0 \mathrm{~s}$, (b) $t=12 \mathrm{~s}$, (c) $t=13 \mathrm{~s}$, and (d) $t=14 \mathrm{~s}$.

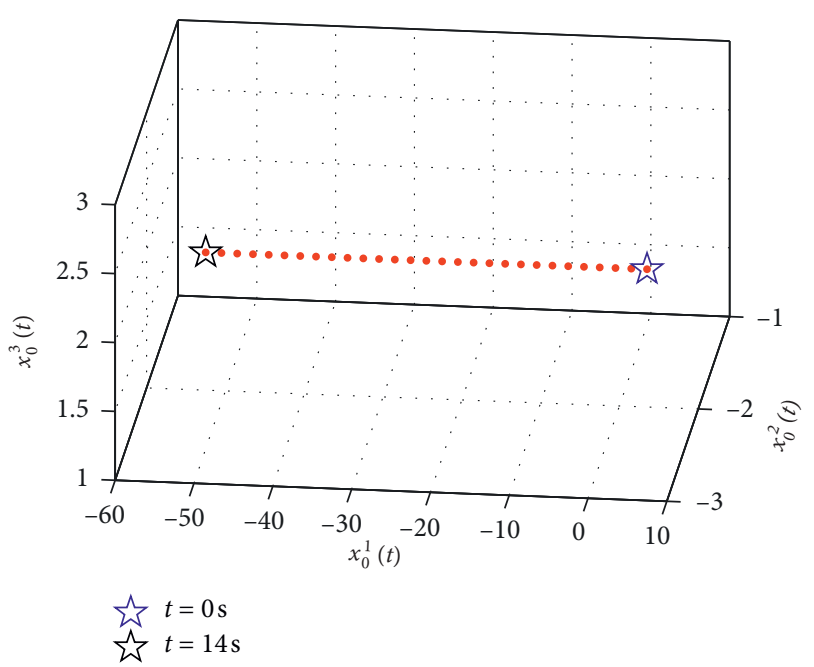

FIgURE 9: Movement track of the leader for time-invariant formation tracking case.

are the trajectories of the collaborative state of the leader. Figure 8 describes the state snapshots of the leader and eight followers at $t=0 \mathrm{~s}, t=12 \mathrm{~s}, t=13 \mathrm{~s}$, and $t=14 \mathrm{~s}$, and the leader and eight followers are depicted by pentagram, asterisks, plusses, circles, $x$-marks, triangles, hexagrams, squares, and diamonds, respectively, where eight followers achieve a regular time-invariant octagon to track the leader. Figure 9 shows that the leader moves along a straight line, where the initial and final states are depicted by a blue pentagram and a black pentagram, respectively. Figure 10 shows the trajectories of the energy consumption and the limited energy. From Figures 7 to 10, one can find that this swam system achieves limited-energy time-invariant formation tracking.

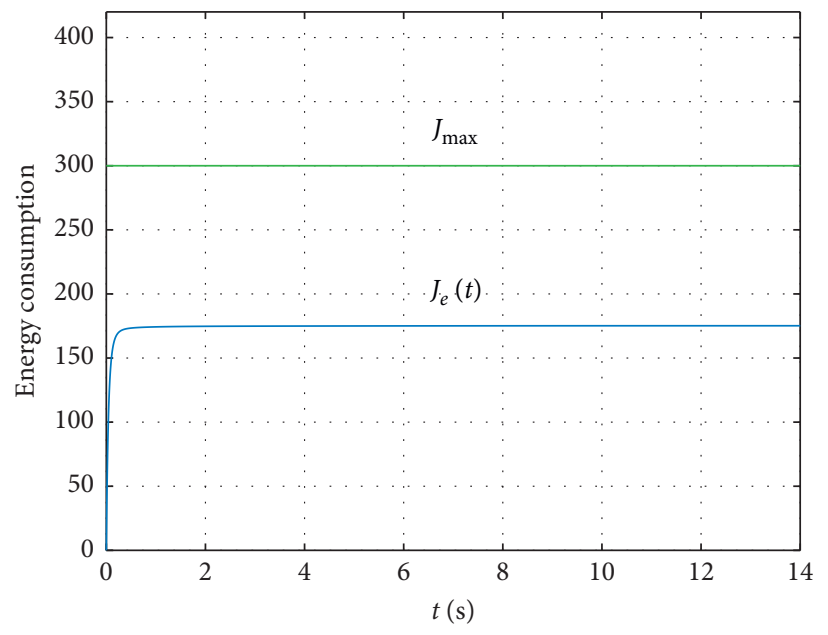

FIGURE 10: Energy consumption and the limited budget for timeinvariant formation tracking case.

\section{Conclusions}

For high-order linear time-invariant swarm systems with the limited energy and fixed topologies, a new formation tracking protocol with an energy integral term was proposed to realize formation tracking under the condition that the whole energy consumption is less than or equal to the limited energy supply. By constructing some nonsingular transformation matrix, the whole dynamics of a swarm system with a leader-following topology structure was divided into two independent parts: the dynamics of the leader and the relative dynamics among all agents, and it was shown that the formation tracking is achieved if the relative dynamics is asymptotically stable. Furthermore, the limited energy was introduced into the formation tracking criteria 
by the matrix variable and disagreement initial states and sufficient conditions for swam systems with the limited energy to achieve time-varying formation tracking and timeinvariant formation tracking were proposed, respectively. Especially, these criteria are independent of the number of agents and only include linear matrix inequality constraints, so they are scalable and checkable; that is, the computation complexity does not increase as the number of agents of a swarm system is added. The further works will focus on extending main conclusions for swarm systems with the homogenous dynamics and the fixed topology to swarm systems with the heterogeneous dynamics and switching topologies.

\section{Data Availability}

The simulation data used to support the findings of this study are included within the article.

\section{Conflicts of Interest}

The authors declare no conflicts of interest.

\section{Authors' Contributions}

Conceptualization was carried out by Jianye Yang and Xinzhong Han. Methodology was developed by Jianye Yang. Cheng Wang was responsible for formal analysis and validation. Investigation and project administration were performed by Hongtao Dang. Writing-original draft preparation and writing-review and editing were carried out by Cheng Wang and Jianye Yang. Xinzhong Han supervised the study. Cheng Wang and Xinzhong Han were involved in funding acquisition. All authors have read and agreed to the published version of the manuscript.

\section{Acknowledgments}

This research was funded by the Key Research and Development Program of Shaanxi (no. 2019GY-025).

\section{References}

[1] R. Olfati-Saber, "Flocking for multi-agent dynamic systems: algorithms and theory," IEEE Transactions on Automatic Control, vol. 51, no. 3, pp. 401-420, 2006.

[2] N. Sun, Y. Fu, T. Yang, J. Zhang, Y. Fang, and X. Xin, "Nonlinear motion control of complicated dual rotary crane systems without velocity feedback: design, analysis, and hardware experiments," IEEE Transactions on Automation Science and Engineering, vol. 17, no. 2, pp. 1017-1029, 2020.

[3] J. Xi, C. Wang, H. Liu, and L. Wang, "Completely distributed guaranteed-performance consensualization for high-order multiagent systems with switching topologies," IEEE Transactions on Systems, Man, and Cybernetics: Systems, vol. 49, no. 7, pp. 1338-1348, 2019.

[4] J. Qu, Z. Ji, C. Lin, and H. Yu, "Fast consensus seeking on networks with antagonistic interactions," Complexity, vol. 2018, Article ID 7831317, 15 pages, 2018.

[5] J. Sun, Z. Geng, Y. Lv, Z. Li, and Z. Ding, "Distributed adaptive consensus disturbance rejection for multi-agent systems on directed graphs," IEEE Transactions on Control of Network Systems, vol. 5, no. 1, pp. 202-212, 2018.

[6] J. Xi, C. Wang, X. Yang, and B. Yang, "Limited-budget output consensus for descriptor multiagent systems with energy constraints," IEEE Transactions on Cybernetics, In press.

[7] Y. Zhang, H. Li, J. Sun, and W. He, "Cooperative adaptive event-triggered control for multiagent systems with actuator failures," IEEE Transactions on Systems, Man, and Cybernetics: Systems, vol. 49, no. 9, pp. 1759-1768, 2019.

[8] J. Xi, L. Wang, J. Zheng, and X. Yang, "Energy-constraint formation for multiagent systems with switching interaction topologies," IEEE Transactions on Circuits and Systems I: Regular Papers, vol. 67, no. 6, pp. 2442-2454, 2020.

[9] X.-G. Guo, J.-L. Wang, F. Liao, and D. Wang, "Quantized $H_{\infty}$ consensus of multiagent systems with quantization mismatch under switching weighted topologies," IEEE Transactions on Control of Network Systems, vol. 4, no. 2, pp. 202-212, 2017.

[10] Z.-Y. Tan, N. Cai, J. Zhou, and S.-G. Zhang, "On performance of peer review for academic journals: analysis based on distributed parallel system," IEEE Access, vol. 7, pp. 19024-19032, 2019.

[11] L. Consolini, F. Morbidi, D. Prattichizzo, and M. Tosques, "Leader-follower formation control of nonholonomic mobile robots with input constraints," Automatica, vol. 44, no. 5, pp. 1343-1349, 2008.

[12] K.-K. Oh and H.-S. Ahn, "Formation control of mobile agents based on distributed position estimation," IEEE Transactions on Automatic Control, vol. 58, no. 3, pp. 737-742, 2013.

[13] H. Liu, T. Ma, F. L. Lewis, and Y. Wan, "Robust formation control for multiple quadrotors with nonlinearities and disturbances," IEEE Transactions on Cybernetics, vol. 50, no. 4, pp. 1362-1371, 2020.

[14] W. Ren, "Consensus strategies for cooperative control of vehicle formations," IET Control Theory \& Applications, vol. 1, no. 2, pp. 505-512, 2007.

[15] Z. Ji, H. Lin, S. Cao, Q. Qi, and H. Ma, "The complexity in complete graphic characterizations of multiagent controllability," IEEE Transactions on Cybernetics, In press.

[16] L. Mo and S. Guo, "Consensus of linear multi-agent systems with persistent disturbances via distributed output feedback," Journal of Systems Science and Complexity, vol. 32, no. 3, pp. 835-845, 2019.

[17] S. Liu, Z. Ji, and H. Ma, "Jordan form-based algebraic conditions for controllability of multiagent systems under directed graphs," Complexity, vol. 2020, Article ID 7685460, 18 pages, 2020.

[18] J. A. Fax and R. M. Murray, "Information flow and cooperative control of vehicle formations," IEEE Transactions on Automatic Control, vol. 49, no. 9, pp. 1465-1476, 2004.

[19] H. Du, G. Wen, Y. Cheng, Y. He, and R. Jia, "Distributed finite-time cooperative control of multiple high-order nonholonomic mobile robots," IEEE Transactions on Neural Networks and Learning Systems, vol. 28, no. 12, pp. 29983006, 2016.

[20] W. Qin, Z. Liu, and Z. Chen, "A novel observer-based formation for nonlinear multi-agent systems with time delay and intermittent communication," Nonlinear Dynamics, vol. 79, no. 3, pp. 1651-1664, 2015.

[21] L. Brinon-Arranz, A. Seuret, and C. Canudas-de-Wit, "Cooperative control design for time-varying formations of multiagent systems," IEEE Transactions on Automatic Control, vol. 59, no. 8, pp. 2283-2288, 2014.

[22] R. Rahimi, F. Abdollahi, and K. Naqshi, “Time-varying formation control of a collaborative heterogeneous multi agent 
system," Robotics and Autonomous Systems, vol. 62, no. 12, pp. 1799-1805, 2014.

[23] X. Dong and G. Hu, "Time-varying formation control for general linear multi-agent systems with switching directed topologies," Automatica, vol. 73, no. 73, pp. 47-55, 2016.

[24] F. Xiao, L. Wang, J. Chen, and Y. Gao, "Finite-time formation control for multi-agent systems," Automatica, vol. 45, no. 11, pp. 2605-2611, 2009.

[25] X. Dong, Y. Zhou, Z. Ren, and Y. Zhong, "Time-varying formation tracking for second-order multi-agent systems subjected to switching topologies with application to quadrotor formation flying," IEEE Transactions on Industrial Electronics, vol. 64, no. 6, pp. 5014-5024, 2017.

[26] X. Dong and G. Hu, "Time-varying formation tracking for linear multiagent systems with multiple leaders," IEEE Transactions on Automatic Control, vol. 62, no. 7, pp. 36583664, 2017.

[27] J. Xi, Z. Fan, H. Liu, and T. Zheng, "Guaranteed-cost consensus for multiagent networks with Lipschitz nonlinear dynamics and switching topologies," International Journal of Robust and Nonlinear Control, vol. 28, no. 7, pp. 2841-2852, 2018.

[28] L. Wang, J. Xi, M. He, and G. Liu, "Robust time-varying formation design for multiagent systems with disturbances: extended-state-observer method," International Journal of Robust and Nonlinear Control, vol. 30, no. 7, pp. 2796-2808, 2020.

[29] J. Yu, X. Dong, Q. Li, and Z. Ren, "Robust $H_{\infty}$ guaranteed cost time-varying formation tracking for high-order multiagent systems with time-varying delays," IEEE Transactions on Systems, Man, and Cybernetics: Systems, vol. 50, no. 4, pp. 1465-1475, 2020.

[30] C. Godsil and G. Royal, Algebraic Graph Theory, Springer, New York, NY, USA, 2001.

[31] S. Boyd, L. E. Ghaoui, E. Feron, and V. Balakrishnan, Linear Matrix Inequalities in System and Control Theory, SIAM, Philadelphia, PA, USA, 1994.

[32] A. Berman and X. D. Zhang, "Lower bounds for the eigenvalues of Laplacian matrices," Linear Algebra and Its Applications, vol. 316, no. 1-3, pp. 13-20, 2000.

[33] R. A. Horn and C. A. Johnson, Matrix Analysis, Cambridge University Press, Cambridge, UK, 1990.

[34] P. Gahinet, A. Nemirovskii, A. J. Laub, and M. Chilali, LMI Control Toolbox User's Guide, The Math Works, Natick, MA, USA, 1995. 